\title{
SUR LES MAXIMA DES FORMES BILINÉAIRES ET SUR LES FONCTIONNELLES LINÉAIRES.
}

\author{
Par
}

MARCEL RIESZ

à Lund.

\section{Introduction.}

1. Il y a vingt ans, JENsen ' a publié dans les Acta mathematica un mémoire important sur les fonctions convexes et les inégalités qui s'y rattachent. Dès lors le rôle des fonctions convexes en l'Analyse est devenu de plus en plus manifeste.

Une fonction $f(P)$, définie pour les points d'un domaine convexe, est dite convexe, si pour deux points quelconques $P_{1}$ et $P_{2} d u$ domaine et pour toutes les valeurs positives $t_{1}$ et $t_{2}$ telles que $t_{1}+t_{2}=\mathrm{I}$, on $a$

$$
f\left(t_{1} P_{1}+t_{2} P_{2}\right) \leqq t_{1} f\left(P_{1}\right)+t_{2} f\left(P_{2}\right)
$$

La signification gelmétrique de cette inégalité est évidente.

2. Cela étant, considérons une forme bilinéaire à un nombre fini de variables

$$
A(x, y)=\sum_{j=1}^{m} \sum_{k=1}^{n} a_{j k} x_{j} y_{k}
$$

à des coefficients quelconques, réels ou non.

Désignons par $M_{\alpha \beta}$ le maximum de $|A(x, y)|$ sous les conditions

$$
\sum_{j=1}^{m}\left|x_{j}\right|^{\frac{1}{\alpha}} \leqq \mathrm{I} ; \quad \sum_{k=1}^{n}\left|y_{k}\right|^{\frac{1}{\beta}} \leqq \mathrm{I} .
$$

1 J. L. W. V. JENSEN, Sur les fonctions convexes et les inégalités entre les valeurs moyennes, Acta math. t. 30 (1906), p. 175-193.

59-2661. Acta mathematica. 49. Imprimé le 11 janvier 1927. 
Dans les cas $\alpha=0$ ou $\beta=0$, les conditions respectives (I) seront, par des raisons de continuité, remplacées par

$$
\left|x_{j}\right| \leqq \mathrm{I} \quad(j=\mathrm{I}, 2, \ldots, m) \quad \text { ou } \quad\left|y_{k}\right| \leqq \mathrm{I} \quad(k=\mathrm{I}, 2, \ldots, n) .
$$

3. Ces préliminaires posés, nous sommes à même d'énoncer le théorème que voici.

I. La fonction $\log M_{\alpha \beta}$ est, dans le triangle

$$
\alpha \leqq \mathrm{I}, \quad \beta \leqq \mathrm{I}, \quad \alpha+\beta \geqq \mathrm{I}
$$

une fonction convexe du point $(\alpha, \beta) .^{1}$

Ce théorème contient en germe tous les résultats du travail présent, en particulier ceux concernant les fonctionnelles linéaires, qui de leur côté embrassent sous un aspect général les théorèmes de MM. W. H. Youna, F. HAUsdorfF ${ }^{9}$ et F. RIesz ${ }^{3}$ sur l'extension de la formule de Parseval et d'autres problèmes de caractère analogue. Il s'agira notamment des fonctions conjuguées et de certaines suites de facteurs attachées aux séries de Fourier et en particulier de telles qui, dans un certain sens, ne changent pas le caractère de la série. Dans la dernière partie de ce travail, en retournant à notre premier théorème, nous discutons la question de la réalité des valeurs des variables qui fournissent le maximum. Nous y verrons qu'il y a une différence essentielle entre le triangle (2) et le triangle complémentaire $\mathrm{O} \leqq \alpha, \mathrm{O} \leqq \beta, \alpha+\beta \leqq \mathrm{I}$. Nous finissons par donner des indications, d'ailleurs peu satisfaisantes, sur la validité de notre théorème dans le carré composé des triangles dont nous venons de parler.

\section{Démonstration du Théorème I.}

4. Nous définirons les nombres $\alpha^{\prime}, a, a^{\prime}$ par les relations

$$
\alpha+\alpha^{\prime}=\mathrm{I}, \quad \alpha a=\mathrm{I}, \quad \alpha^{\prime} a^{\prime}=\mathrm{I}
$$

c'est-à-dire que

1 Il en ressort que $M_{\alpha \beta}$ et toutes ses puissances à des exposants positifs sont anssi convexes.

2 F. Hausdorff, Eine Ausdehnung des Parsevalschen Satzes über Fourierreihen, Math. Zeitschr. t. I6 (1923), p. I63-I69. On y trouve une liste des travanx relatifs de M. Youxa.

( F. RIEsz, Utber eine Verallgemeinerung der Parsevalschen Formel, Math. Zeitschr. t. 18 (I923), p. II7-I24. 
Sur les maxima des formes bilinéaires et sur les fonctionnelles linéaires. 467

$$
\alpha^{\prime}=\mathrm{I}-\alpha, \quad a=\frac{\mathrm{I}}{\alpha}, \quad a^{\prime}=\frac{\mathrm{I}}{\alpha^{\prime}}=\frac{\mathrm{I}}{\mathrm{I}-\alpha}=\frac{a}{a-\mathrm{I}} .
$$

Notations analogues pour la lettre $\beta$ et pour d'autres lettres.

On admettra toujours

$$
\circ \leqq \alpha \leqq \mathrm{I}, \quad \circ \leqq \beta \leqq \mathrm{I} ; \quad a \geqq \mathrm{I}, \quad b \geqq \mathrm{I} .
$$

La démonstration de notre théorème se fonde entièrement sur l'inégalité connue de HöLDER ${ }^{1}$

$$
\left|\sum_{j=1}^{m} \xi_{j} \eta_{j}\right| \leqq\left(\sum_{j=1}^{m}\left|\xi_{j}\right|^{a}\right)^{\alpha}\left(\sum_{j=1}^{m}\left|\eta_{j}\right|^{n^{\prime}}\right)^{\alpha^{\prime}}
$$

qui résulte immédiatement de ce que $x^{a}$ est, pour $a>$ I et pour $x \geqq 0$, une fonction convexe de $x$. On peut encore préciser cette inégalité en rappelant le fait que le signe d'égalité intervient seulement dans le cas où le rapport $\left|\xi_{j}\right|^{n}:\left|\eta_{j}\right|^{a^{\prime}}$ et l'amplitude du produit $\xi_{j} \eta_{j}$ sont indépendants de $j$.

On aura encore besoin du cas particulier suivant de (4):

$$
\sum_{j=1}^{m}\left|x_{j}\right|^{h_{1} \sigma_{1}+h_{2} \sigma_{2}} \leqq\left(\sum_{j=1}^{m}\left|x_{j}\right|^{h_{1}}\right)^{\sigma_{1}}\left(\sum_{j=1}^{m}\left|x_{j}\right|^{h_{2}}\right)^{\sigma_{2}}
$$

où $\mathrm{o} \leqq \sigma_{1}, o \leqq \sigma_{2}, \sigma_{1}+\sigma_{2}=\mathrm{I}, h_{1}$ et $h_{2}$ étant des nombres réels quelconques.

5. En posant

$$
X_{k}=\sum_{j=1}^{m} a_{j k} x_{j}(k=\mathrm{I}, 2, \ldots, n) ; \quad Y_{j}=\sum_{k=1}^{n} a_{j k} y_{k}(j=\mathrm{I}, 2, \ldots, m)
$$

on pourra écrire

$$
A(x, y)=\sum_{j=1}^{m} x_{j} Y_{j}=\sum_{k=1}^{n} y_{k} X_{k}
$$

En écrivant alors les conditions (I) sous la forme

(7)

$$
\sum_{j=1}^{n}\left|x_{j}\right|^{a} \leqq \mathrm{I} ; \quad \sum_{k=1}^{n}\left|y_{k}\right|^{b} \leqq \mathrm{I}
$$

1 o. HöLDER, Ueber einen Mittelwerthssatz, Götting. Nachr. 1889, p. 38-47. Cf. aussi JENSEN, l. c. p. 182. 
nous allons montrer que l'expression

$$
\left(\sum_{k=1}^{n}\left|X_{k}\right|^{\prime}\right)^{\beta^{\prime}}
$$

variée sous la première des conditions (7), et l'expression

$$
\left(\sum_{j=1}^{m}\left|Y_{j}\right|^{a^{\prime}}\right)^{\alpha^{\prime}}
$$

varièe sous la seconde de ces conditions, admettent aussi $M_{\alpha \beta}$ comme maximum. On, en se débarrassant des conditions (7), on pourra dire:

\section{Les rapports}

$$
|A(x, y)|:\left(\sum_{j=1}^{m}\left|x_{j}\right|^{a}\right)^{\alpha}\left(\sum_{k=1}^{n}\left|y_{k}\right|^{b}\right)^{\beta}
$$

$$
\left(\sum_{k=1}^{n}\left|X_{k}\right|^{b^{\prime}}\right)^{\beta^{\prime}}:\left(\sum_{j=1}^{m}\left|x_{j}\right|^{a}\right)^{\alpha}
$$

$$
\left(\sum_{j=1}^{m}\left|Y_{j}\right|^{a^{\prime}}\right)^{\alpha^{\prime}}:\left(\sum_{k=1}^{n}\left|y_{k}\right|^{b}\right)^{\beta}
$$

admettent le même maximum $\boldsymbol{M}_{\alpha \beta}$.

En effet, on a d'abord, en appliquant l'inégalité de Hölder à (6),

$$
|A(x, y)| \leqq\left(\sum\left|Y_{j}\right|^{a^{\prime}}\right)^{\alpha^{\prime}}\left(\sum\left|x_{j}\right|^{a}\right)^{\alpha}
$$

C'est-à-dire que le rapport (9) ne pourra dépasser le rapport (IO').

D'autre part, d'après la remarque dont on a fait suivre l'inégalité de Hölder, on pourra, pour des $y_{k}$ arbitraires, déterminer les $x_{j}$ de façon qu'il $y$ ait égalité entre les deux membres de (II), et alors aussi les rapports (9) et (10') seront égaux. Il y a donc égalité entre les maxima de (9) et de (10') et dès lors, par symétrie, aussi entre ceux de (9) et de (Io).

6. Montrons maintenant que tout système $\left(x_{j} ; y_{k}\right)$ qui rend $|A(x, y)|$ maximum, sous les conditions (7), satisfait aux relations

$$
\begin{cases}\left|Y_{j}\right|=M_{\alpha \beta}\left|x_{j}\right|^{a-1} & (j=\mathrm{I}, 2, \ldots, m) ; \\ \left|X_{k}\right|=M_{\alpha \beta}\left|y_{k}\right|^{b-1} & (k=\mathrm{I}, 2, \ldots, n) .\end{cases}
$$


Sur les maxima des formes bilinéaires et sur les fonctionnelles linéaires.

En effet, désignons un instant par $\mu$ le maximum de $|A(x, y)|$ sous la première des conditions (7), les $y_{k}$ étant fixés. L'inégalité (I I) et notre remarque ajoutée à l'inégalité de Hölder montrent que pour que ce maximum soit atteint, il faudra choisir les $x_{j}$ de façon que les rapports

$$
\left|Y_{j}\right|^{a^{\prime}}:\left|x_{j}\right|^{a}=\left|Y_{j}\right|^{\frac{a}{a-1}}:\left|x_{j}\right|^{a}
$$

et alors les rapports

$$
\left|Y_{j}\right|:\left|x_{j}\right|^{a-1}=-\lambda
$$

soient indépendants de $j$ et qu'il en soit de même des amplitudes des $x_{j} Y_{j}$. Il vient

$$
\mu=\left|\sum x_{j} Y_{j}\right|=\sum\left|x_{j}\right|\left|Y_{j}\right|=\lambda \sum\left|x_{j}\right|^{a}=\lambda
$$

et alors on aura les équations

$$
\left|Y_{j}\right|==\mu\left|x_{j}\right|^{a-1}
$$

Or, si l'on considère un système $\left(x_{j} ; y_{k}\right)$ qui rend $|A(x, y)|$ maximum sous les conditions $(7)$, ce système rendra encore $|A(x, y)|$ maximum, si l'on fixe les $y_{k}$ et ne fait varier que les $x_{j}$. Alors le premier système des équations (12) se trouve établi. Le second système en résulte par symétrie.

7. Cela étant, désignons par $(\alpha, \beta),\left(\alpha_{1}, \beta_{1}\right),\left(\alpha_{2}, \beta_{2}\right)$ trois points du triangle (2), situés en ligne droite, le point $(\alpha, \beta)$ séparant les deux autres. Posons encore pour abréger $M_{\alpha \beta}=M, M_{\alpha_{1} \beta_{1}}=M_{1}$ et $M_{\alpha_{*} \beta_{2}}=M_{2}$. Notre théorème revient alors à l'inégalité

$$
M \leqq M_{1}^{\frac{\alpha_{2}-\alpha}{\alpha_{2}-\alpha_{1}}} M_{2}^{\frac{\alpha-\alpha_{1}}{\alpha_{2}-\alpha_{1}}}=M_{1}^{t_{1}} M_{2}^{t_{2}}
$$

où $t_{1}+t_{\mathbf{2}}=\mathrm{I}$.

Or, des relations ( 12 ) et de ce qu'on a dit au sujet du maximum de (Io) et de (Io'), on obtient immédiatement que tout système $\left(x_{j} ; y_{k}\right)$ qui fournit le maximum de $|A(x, y)|$, sous les conditions (7), satisfait aux inégalités

$$
\begin{aligned}
& M\left(\sum\left|x_{j}\right|^{(a-1) a_{2}^{\prime}}\right)^{\alpha_{1}^{\prime}}=\left(\sum\left|Y_{j}\right|^{\alpha_{1}^{\prime}}\right)^{\alpha_{1}^{\prime}} \leqq M_{1}\left(\sum\left|y_{k}\right|^{b_{1}}\right)^{\beta_{1}} ; \\
& M\left(\sum\left|y_{k}\right|^{(b-1) b_{z^{\prime}}}\right)^{\beta_{2}{ }^{\prime}}=\left(\sum\left|X_{k}\right|^{b_{z^{\prime}}}\right)^{\beta_{z^{\prime}}} \leqq M_{2}\left(\sum\left|x_{j}\right|^{a_{2}}\right)^{\alpha_{2}} .
\end{aligned}
$$

De là 


$$
\begin{aligned}
& M\left(\sum\left|x_{j}\right|^{(a-1) a_{2}{ }^{\prime}}\right)^{\alpha_{1} t_{1}}\left(\sum\left|y_{k}\right|^{(b-1) b_{2}{ }^{\prime}}\right)^{\beta_{2}^{\prime} t_{2}} \leqq \\
& \leqq M_{1}^{t_{1}} M_{2}^{t_{2}}\left(\sum\left|x_{j}\right|^{a_{2}}\right)^{\alpha_{2} t_{2}}\left(\sum\left|y_{k}\right|^{b_{1}}\right)^{\beta_{1} t_{1}} \cdot 1
\end{aligned}
$$

Déterminons maintenant $\lambda$ et $\mu$ de façon $q u e^{2}$

$$
a_{2}=\lambda(a-\mathrm{I}) a_{1}^{\prime}+(\mathrm{I}-\lambda) a ; \quad b_{1}=\mu(b-\mathrm{I}) b_{2}^{\prime}+(\mathrm{I}-\mu) b
$$

On trouve par un calcul simple

$$
\lambda=\frac{\alpha_{1}^{\prime} \frac{\alpha_{9}-\alpha}{\alpha}}{\alpha_{2}} \frac{\alpha-\alpha_{1}}{\alpha-\beta_{2}{ }^{\prime}} ; \quad \mu=\frac{\beta-\beta_{1}}{\beta_{1} \frac{\beta_{2}-\beta}{\beta_{2}-\beta}}=\frac{\alpha-\alpha_{1}}{\beta_{1}} \frac{\beta_{2}-\alpha}{\alpha_{2}}
$$

Admettons un instant que les points $(\alpha, \beta),\left(\alpha_{1}, \beta_{1}\right),\left(\alpha_{2}, \beta_{2}\right)$ soient tels que les valeurs $\lambda$ et $\mu$, qui sont toujours positives, soient aussi $\leqq$ I. Alors on pourra poser dans $\left(4^{\prime}\right), h_{1}=(a-\mathrm{I}) a_{1}^{\prime}, h_{2}=a, \sigma_{1}=\lambda, \sigma_{2}=1-\lambda$ et, en remplaçunt les $x_{j}$ par les $y_{k}, h_{1}=(b-\mathrm{I}) b_{2}{ }^{\prime}, h_{2}=b, \sigma_{1}=\mu, \sigma_{2}=\mathrm{I}-\mu . \quad$ Il viendra alors, en tenant encore compte des inégalités ( 7$)$,

$$
\sum\left|x_{j}\right|^{a_{2}} \leqq\left(\sum\left|x_{j}\right|^{(a-1) a_{1}{ }^{\prime}}\right)^{\lambda} ; \quad \sum\left|y_{k}\right|^{b_{2}} \leqq\left(\sum\left|y_{k}\right|^{(b-1) b_{2}^{\prime}}\right)^{\mu}
$$

et de là, grâce aux relations faciles à vérifier $\lambda \alpha_{2} t_{2}=\alpha_{1}{ }^{\prime} t_{1}, \mu \beta_{1} t_{1}=\beta_{2}{ }^{\prime} t_{2}$,

$$
\left(\sum\left|x_{j}\right|^{a_{2}}\right)^{\alpha_{2} t_{2}} \leqq\left(\sum\left|x_{j}\right|^{(a-1) a_{2}^{\prime}}\right)^{\alpha_{1}^{\prime} t_{1}} ; \quad\left(\sum\left|y_{k}\right|^{b_{1}}\right)^{\beta_{1} t_{1}} \leqq\left(\sum\left|y_{k}\right|^{(b-1) b_{2}^{\prime}}\right)^{\beta_{2}^{\prime} t_{2}}
$$

Par là, l'inégalité ( $3^{\prime}$ ) se réduit à l'inégalité (1 3 ).

8. Tout revient donc à examiner les inégalités

$$
\lambda=\frac{\alpha_{1}^{\prime}}{\alpha_{2}} \frac{\alpha_{2}-\alpha}{\alpha-\alpha_{1}} \leqq \mathrm{I} ; \quad \mu=\frac{\beta_{2}^{\prime}}{\beta_{1}} \frac{\alpha-\alpha_{1}}{\alpha_{2}-\alpha} \leqq \mathrm{I},
$$

ou les inégalités équivalentes

1 Jusqu'ici il aurait suffi de supposer que nos points se trouvent dans le carré $0 \leqq \alpha \leqq$ I, $0 \leqq \beta \leqq I$.

2 $C f$. pour un calcul analogue F. HAUsdorfF et F. RIEsz, l. $c$. 
Sur les maxima des formes bilinéaires et sur les fonctionnelles linéaires. 471

$$
\frac{\alpha_{1}^{\prime}}{\alpha_{2}} \leqq \frac{\alpha-\alpha_{1}}{\alpha_{2}-\alpha} \leqq \frac{\beta_{1}}{\beta_{2}{ }^{\prime}}
$$

Remarquons d'abord, que si les points $\left(\alpha_{1}, \beta_{1}\right)$ et $\left(\alpha_{2}, \beta_{2}\right)$ sont intérieurs au triangle (2), on a

$$
0<\alpha_{1}^{\prime}=\mathrm{I}-\alpha_{1}=\mathrm{I}-\left(\alpha_{1}+\beta_{1}\right)+\beta_{1}<\beta_{1},
$$

et, d'une manière analogue,

$$
0<\beta_{2}^{\prime}<\alpha_{2}
$$

Il s'ensuit que

$$
0<\frac{\alpha_{1}^{\prime}}{\alpha_{2}}<\frac{\beta_{1}}{\beta_{\Omega}^{\prime}}<+\infty .
$$

D'autre part en faisant varier $\alpha$ de $\alpha_{1}$ à $\alpha_{z}$, le rapport $\left(\alpha-\alpha_{1}\right):\left(\alpha_{2}-\alpha\right)$ ira d'une manière continue de o à $+\infty$; on pourra donc choisir $\alpha$ entre $\alpha_{1}$ et $\alpha_{2}$ de sorte que les inégalités (I4) soient remplies. C'est-à-dire que le calcul du numéro précédent et l'inégalité ( $\left.{ }_{1} 3\right)$ sont valables pour les extrémités $\left(\alpha_{1}, \beta_{1}\right)$, $\left(\alpha_{2}, \beta_{2}\right)$ d'un segment de droite quelconque intérieur au triangle (2) et pour certains points intermédiaires $(\alpha, \beta)$ du segment.

Cela signifie en langage géométrique que la surface qui, pour $(\alpha, \beta)$ intérieur au triangle (2), représente la fonction évidemment continue $\log M_{\alpha \beta}$, est telle que aucune de ses cordes n'est située entièrement au-dessous de la surface. Je dis que la surface est convexe. En effet, dans le cas contraire, il existerait un segment de droite intérieur an triangle (2) et tel que l'are de courbe qui y correspond sur la surface, contienne au moins un point situé audessus de la corde joignant les extrémités de l'arc en question. En partant de ce point et en cheminant sur l'arc dans les deux directions jusqu'à la première rencontre avec la corde, on aura construit un are situé entièrement au-dessus de sa corde, ce qui serait en contradiction avec le fait que nous venons d'établir. Notre théorème étant démontré pour l'intérieur du triangle (2), le passage au triangle fermé se fait par continuité. ${ }^{1}$

\section{Seconde forme du théorème I et généralisation.}

9. Dans les applications, il sera souvent préférable de considérer, au lieu de la forme bilinéaire $A(x, y)$, les substitutions linéaires, définies par les formu-

${ }^{1}$ Le raisonnement qui précède tient aussi sans passage à la límite ponr les points $\alpha+\beta=1$, $\alpha>0, \beta>0$. 
les (5), qui y correspondent. On pourra, par des raisons de symétrie, se borner à la substitution $\left(X_{k}\right)$ portant sur les $\left(x_{j}\right)$. On a vu que l'expression (8), variée sous la première des conditions (I), admet le même maximum que $|A(x, y)|$, variée sous ces conditions (I). De là le théorème, où nous avons posé $\gamma$ au lieu de $\beta^{\prime}=\mathrm{x}-\boldsymbol{\beta}$.

II. En désignant par $M_{\alpha \gamma}^{*}$ le maximum de l'expression

$$
\left(\sum_{k=1}^{n}\left|X_{k}\right|^{\frac{1}{\gamma}}\right)^{\gamma}
$$

sous la condition

$$
\sum_{j=1}^{m}\left|x_{j}\right|^{\frac{1}{\alpha}} \leqq \mathrm{I}
$$

$\log M_{\alpha \gamma}^{*}$ sera, dans le triangle

$$
0 \leqq \gamma \leqq \alpha \leqq 1,
$$

une fonction convexe du point $(\alpha, \gamma)$.

10. Voici maintenant une généralisation du théorème $I$, qui se démontre par un calcul analogue, où le rôle des formes linéaires $X_{k}$ et $Y_{j}$ est joué par les formes $\frac{X_{k}}{\sigma_{k}}$ et $\frac{Y_{j}}{\varrho_{j}}$.

I'. Le théorème I reste valable lorsqu'on remplace les conditions (I) par les conditions

$$
\sum_{j=1}^{m} \varrho_{j}\left|x_{j}\right|^{\frac{1}{\alpha}} \leqq \mathrm{I} ; \quad \sum_{k=1}^{n} \sigma_{k}\left|y_{k}\right|^{\frac{1}{\beta}} \leqq \mathrm{I},
$$

les $\varrho_{j}$ et les $\sigma_{k}$ désignant des nombres positifs.

La généralisation correspondante du théorème II s'obtient en appliquant le théorème précédent à la forme bilinéaire $\Sigma \sigma_{k} y_{k} X_{k}$.

II'. En désignant par $\boldsymbol{M}_{\alpha}^{*}$ le maximum de l'expression

$$
\left(\sum_{k=1}^{n} \sigma_{k}\left|X_{k}\right|^{\frac{1}{\gamma}}\right)^{\gamma}
$$

sous la condition

$$
\sum_{j=1}^{m} \rho_{j}\left|x_{j}\right|^{\frac{1}{\alpha}} \leqq \mathrm{I}
$$

$\log M_{\alpha \gamma}^{*}$ sera, dans le triangle (15), une fonction convexe du point $(\alpha, \gamma)$. 
Sur les maxima des formes bilinéaires et sur les fonctionnelles linéaires. 473

\section{Une application.}

11. Avant d'aller aux applications exigeant des passages à la limite, déduisons du théorème II le théorème suivant dû à M. F. RIFsz: ${ }^{1}$

Soit

$$
X_{k}=\sum_{j=1}^{m} a_{j k} x_{j} \quad(k=\mathrm{I}, 2, \ldots, m),
$$

une substitution orthogonale et normée, c'est-à-dive une substitution telle que pour. tout système $\left(x_{j}\right)$ on ait

$$
\sum_{k-1}^{m}\left|X_{k}\right|^{2}=-\sum_{j=1}^{m}\left|x_{j}\right|^{2}
$$

Désignons encore par $\mathfrak{M}$ la plus grande des valeurs $\left|a_{j k}\right|$. Alors on aura pour tout nombre a tel que $\mathrm{I} \leqq a \leqq 2$,

$$
\left(\sum_{k=1}^{m}\left|X_{k}\right|^{-a}{ }^{a-1}\right)^{\frac{a-1}{a}} \leqq \mathfrak{M}^{2-a} \frac{a}{a}\left(\sum_{j=1}^{m}\left|x_{j}\right|^{a}\right)^{1}
$$

Posons, comme d'habitude, $\frac{\mathrm{I}}{a}=\alpha$ et considérons, dans le plan $(\alpha, \gamma)$, le segment de droite qui est limité par les points $(I, 0)$ et $\left(\frac{1}{2}, \frac{1}{2}\right)$ et qui, évidemment, satisfait à l'équation $\gamma=\mathrm{I}-\alpha$. Ce segment étant situé dans le triangle $o \leqq \gamma \leqq \alpha \leqq I$, on pourra $\mathrm{y}$ appliquer le théorème II. Or on a évidemment $M_{\frac{1}{2}, \frac{1}{2}}^{*}=\mathrm{I}$. De plus on a $M_{1,0}^{*}=-$ Mi. En effet, $\left(c f\right.$. le $\left.\mathbf{n}^{\circ} 2\right) M_{1,0}^{*}$ sera égal à la plus grande valeur que pourra atteindre l'une ou l'autre des $\left|X_{k}\right|$, variées sous la condition $\Sigma\left|x_{j}\right|=\mathrm{I}$, c'est-à-dire à la plus grande des valeurs $\left|a_{i k}\right|$. Il viendra donc pour tout point $(\alpha, \gamma)$ situé sur ledit segment

$$
M_{\alpha \gamma}^{*} \leqq\left(M_{1,0}^{*}\right)^{\frac{\alpha-\frac{1}{2}}{1-\frac{1}{2}}}\left(M_{\frac{1}{2}, \frac{1}{2}}^{*}\right)^{\frac{1}{1-\frac{1}{2}}}=\mathfrak{M}^{2 \alpha-1}=\mathfrak{M}^{2-a}
$$

On voit que l'orthogonalité intervient très peu et que le théorème subsiste dans tous les cas où l'on a

${ }^{1}$ l. c. p. 124. Nous reviendrons plus loin sur la généralisation da théorème de Parseval donnée dans le travail cité et dont le théorème du texte est un cas particulier. M. F. RIEsz a observé que ce théorème particulier fournit de son côté, par un passage à la limite, la généralisation en question du théorème de Parseval.

60-2661. Acta malhematica. 49. Imprimé lo 11 janvier 1027. 


$$
\sum_{k=1}^{n}\left|X_{k}\right|^{2} \leqq \sum_{j=1}^{m}\left|x_{j}\right|^{2},
$$

c'est-à-dire où la borne de la forme bilinéaire $A(x, y)$, prise dans le sens classique, est $\leqq I$.

\section{Opérations bilinéaires et transformations linéaires.}

12. En quittant maintenant le point de vue algébrique et en laissant aux soins du lecteur l'extension presqu'immédiate de nos résultats aux formes bilinéaires et aux substitutions linéaires à une infinité de variables, nous passons aux applications à l'Analyse fonctionnelle.

Rappelons d'abord quelques faits connus concernant l'intégrale de StieltjesLebesgue et les opérations fonctionnelles. ${ }^{1}$ Ce n'est que pour fixer les idées que nous nous bornons à des fonctions d'une seule variable, nos résultats s'étendant immédiatement à des champs plus généraux. Pour ces champs, on se servira avec avantage de l'idée de fonctions d'ensemble.

Envisageons alors une intégrale de Stieltjes-Lebesgue de la forme

$$
\int_{x}^{\lambda} f(x) d \varphi(x)
$$

$\varphi(x)$ étant une fonction non décroissante définie daus l'intervalle $x \leqq x \leqq \lambda$.

Dans les applications particulières de nos résultats, $\varphi(x)$ sera ou bien identique à la variable indépendante $x$, ou bien $\varphi(x)$ sera constante par intervalles, ne croissant que par des sauts brusques. Dans le premier cas, il s'agira tout simplement de l'intégrale ordinaire de Lebesgue $\int f(x) d x$, dans le second, l'intégrale de Stieltjes-Lebesgue se réduira à une somme de la forme $\Sigma \varrho_{k} f\left(x_{k}\right)$,

1 Pour ces faits et pour les indications bibliographiques voir entre autres: J. RaDoN, Theorie und Anwendungen der absolut additiven Mengenfunktionen, Sitzungsber. Akad. Wien, t. 122, Abt. II a, (1913), p. I 295-1438; T. H. Hildebrandt, On integrals related to and extensions of the Lebesgue integrals, Am. M. S. Bull. t. 24 (I918), p. I13-I44, I77-202. C. DE IAA VALLEE Poussix, Les fonctions à variation bornée et les questions qui s'y rattachent, Bull. des Sciences. Math. (2) t. 44 (1920), p. 267-296.

Dans toutes les applications qui suivent, même dans celles concernant des séries, on pourrait s'arranger arec l'intégrale de Lebesgue, mais nous préférons exposer nos résultats dås le langage de l'intégrale plus générale de Stieltjes-Lebesgue, qui permet d'une manière naturelle de considérer séries et intégrales sous le même point de vue. 
Sur les maxima des formes bilinéaires et sur les fonctionnelles linéaires. 475

les $x_{k}$ étant les points de discontinuité de $\varphi(x)$ et les $\varrho_{k}$ les sauts correspondants. On voit que, dans ce second cas, ce ne sont que les valeurs particulières prises par la fonction $f(x)$ aux points $x_{k}$ qui interviennent dans l'intégrale.

Dans le cas général, l'intégrale ci-dessus se définit de la manière suivante. On considère la courbe $y=\varphi(x)$ et on forme la fonction inverse $x=x(y)$, avec des conventions évidentes pour les valeurs de $x$ et de $y$ qui correspondent à des segments horizontaux ou verticaux ${ }^{1}$ de la courbe. En posant alors $f(x)=$ $f(x(y))=h(y),{ }^{2}$ l'intégrale ci-dessus se transforme dans une intégrale de la forme $\int_{\tau}^{\omega} h(y) d y$. Si cette dernière intégrale existe au sens de M. Lebesgue, on dira que $f(x)$ est intégrable par rapport à $\varphi(x)$ et on posera par définition

$$
\int_{x}^{\lambda} f(x) d \varphi(x)=\int_{i}^{\omega} h(y) d y
$$

13. On dira que la fonction $f(x)$, définie dans le même intervalle que $\varphi(x)$, appartient à la classe $L_{\varphi}^{a}(a \geqq \mathrm{I})$, si $\int f(x) d \varphi(x)$ existe dans tout intervalle où la variation de $\varphi(x)$ est finie et si l'intégrale

$$
\int_{x}^{\lambda}|f(x)|^{a} d \varphi(x)
$$

existe.

Cela étant, considérons encore une seconde fonction non décroissante $\psi(x)$, définie dans un certain intervalle $\mu \leqq x \leqq \nu$, et la classe $L_{\psi}^{b}(b \geqq \mathrm{I})$ qui y correspond.

Dans les développements qui suivent, les fonctions $\varphi(x)$ et $\psi(x)$, une fois définies, resteront toujours les mêmes.

14. Envisageons maintenant à la fois les classes $L_{q}^{a}$ et $L_{\psi}^{b}$ et faisons correspondre à chaque couple de fonctions $f(x)$ et $g(x)$ tirées des classes respectives un nombre $A(f, g)$ de sorte que les hypotheses suivantes soient remplies.

1 A toutes les valeurs de $y$ appartenant à un segment vertical, il correspond nne seule valeur de $x$, l'abscisse de tons les points du segment; à une valeur de $y$ appartenant à un segment horizontal, on fait correspondre une quelconque des abscisses des points du segment.

' La fonction $h(y)$ sera, en général, indéterminée pour les valeurs de $y$ qui correspondent à des segments horizontaux; cependant ces valeurs formant un ensemble dénombrable, n'exerceront aucune influence sur l'intégrale, si l'on la prend au sens de Lebesgue. 
a) La correspondance est distributive, c'est-à-dire que

$$
A\left(f_{1}+f_{2}, g_{1}+g_{2}\right)=A\left(f_{1}, g_{1}\right)+A\left(f_{1}, g_{2}\right)+A\left(f_{2}, g_{1}\right)+A\left(f_{2}, g_{2}\right)
$$

et

$$
A(\lambda f, \mu g)=\lambda \mu A(f, g)
$$

où $\lambda$ et $\mu$ désignent des constantes arbitraires;

b) elle est bornée, c'est qu'il existe un nombre $M$ tel que l'on ait toujours

$$
|A(f, g)| \leqq M\left(\int|f|^{a} d \varphi\right)^{\frac{1}{a}}\left(\int|g|^{b} d \psi\right)^{\frac{1}{b}} \cdot 1
$$

Sous ces hypothèses $A(f, g)$ sera dite une opération bilinéaire portant sur le $\operatorname{champ}\left(L_{\varphi}^{a}, L_{\psi}^{b}\right)$. Le plus petit nombre $M$ qui suffit pour que l'inégalité (2o) soit remplie, sera appelé la borne de l'opération bilinéaire.

On dira qu'une opération bilinéaire $A$ porte sur plusieurs champs, si elle est définie pour tous ces champs, et cela d'une façon univoque pour les éléments qui appartiennent à la fois à deux ou à plusieurs de ces champs. La borne de l'opération dépendra alors des exposants $a$ et $b$, caractérisant les champs, et c'est précisément cette dépendance que nous étudierons dans la suite.

15. Il résulte immédiatement de l'inégalité (20) qu'une opération bilinéaire portant sur un certain champ $\left(L_{\uparrow,}^{a}, L_{\psi}^{b}\right)$ est déterminée d'une manière univoque lorsqu'on connaît ses valeurs pour les éléments de deux ensembles de fonctions tirés des deux classes $L_{\varphi}^{a}$ et $L_{\psi}^{b}$, partout denses dans les classes respectives, l'écart de deux fonctions $f_{1}$ et $f_{q}$ ou $g_{1}$ et $g_{2}$ étant toujours mesuré par

$$
\left(\int\left|f_{1}-f_{2}\right|^{a} d \varphi\right)^{\frac{1}{a}} \text { ou }\left(\int\left|g_{1}-g_{2}\right|^{b} d \psi\right)^{\frac{1}{b}}
$$

respectivement. Mais il y en a plus. Admettons qu'on ne définisse d'abord une opération que pour les éléments de deux ensembles tels qu'on vient de dire et qui, en outre, contiennent les combinaisons linéaires de leurs éléments. Alors, si les hypothèses a) et b) sont satisfaites pour les éléments desdits ensembles, l'opération se définit immédiatement par continuité dans tout le champ $\left(L_{\varphi}^{a}, L_{\psi}^{b}\right)$, la borne

1 Dans la suite, pour simplifier l'écriture, nous supprimerons, en général, la variable et les limites d'intégration.

'Ce n'est que pour des raisons de rédaction que nous traitons les opérations bilinéaires avant d'avoir parlé des opérations linéaires. 
Sur les maxima des formes bilinéaires et sur les fonctionnelles linéaires.

étant la même pour tout le champ que celle qui correspond à nos deux ensembles particuliers.

16. Un exemple presqu'évident d'ensembles particuliers tels qu'on vient de dire est fourni par les fonctions constantes par intervalles, ne présentant qu'un nombre fini de sauts et s'annulant dans ceux des intervalles en question où la variation respective de $\varphi(x)$ ou de $\psi(x)$ est infinie. ${ }^{1}$

Sur bien d'autres exemples qu'il serait facile de former, le susdit a l'avantage de faire immédiat le passage aux formes bilinéaires à un nombre fini de variables dont nous allons parler tout à l'heure. D'autre part, ces fonctions appartenant à tous les champs $\left(I_{\psi}^{a}, L_{\psi}^{b}\right)$, on pourra les utiliser pour étendre l'opération en passant d'un champ à un autre. Tout dépend alors de savoir si l'opération appliquée à ces fonctions particulières possède une borne finie par rapport aux exposants variés. S'il en est ainsi, on fera l'extension en passant par continuité de nos fonctions au champ complet.

17. En désignant, comme plus haut pour les formes bilinéaires, la borne correspondant aux exposants $a$ et $b$ par $M_{\alpha \beta}{ }^{2}$ où $\alpha=\frac{\mathrm{I}}{a}, \beta=\frac{\mathrm{I}}{b}$, nous sommes à même d'énoncer le théorème suivant, extension des théorèmes $I$ et $I^{\prime}$.

III. Soit $A$ une opération bilinéaire portant sur tous les champs $\left(L_{\varphi}^{a}, L_{\psi}^{b}\right) q u i$ correspondent aux points $\left(\alpha=\frac{\mathrm{I}}{a}, \beta=\frac{\mathrm{I}}{b}\right)$ d'un segment de droite appartenant au triangle (2), alors $\log M_{\alpha \beta}$ est une fonction convexe des points $(\alpha, \beta)$ du segment en question.

La convexité de la fonction $\log M_{\alpha \beta}$ suggère encore le théorème suivant qu'on va démontrer en même temps.

IY. Chaque fois que l'opération est définie pour les champs correspondant $\grave{a}$ deux points du triangle (2), elle pourra être étendue $\grave{a}$ tous les champs correspondant aux points du segment joignant les deux points donnés, cette extension étant déterminée d'une manière univoque.

Admettons d'abord les hypothèses du théorème III. Considérons alors les ensembles de fonctions $f$ et $g$, constantes par intervalles et telles qu'on vient de dire, les intervalles étant fixés jusqu'à nouvel ordre. En numérotant ces inter-

${ }^{1}$ Evidemment, cela ne pourra arriver que dans les intervalles extrêmes.

2 Dans la définition de $M_{0 \beta}$, on aura à remplacer dans le second membre de (20) le facteur correspondant à $f$ par la borne supérieure de $|f|$ au sens de Lebesgue, c'est-à-dire par le plus petit nombre que $|f|$ ne dépasse que dans un ensemble nal an plus. La définition de $M_{\alpha 0}$ se fait d'ane manière analogue. 
valles et en désignant les valeurs respectives de $f(x)$ et de $g(x)$ par $x_{1}, x_{2}, \ldots, x_{m}$ et $y_{1}, y_{2}, \ldots, y_{n}$, l'opération $A(f, g)$ devient, pour ces fonctions particulières, une forme bilinéaire, tandis que les intégrales $\int|f|^{a} d \varphi$ et $\int|g|^{b} d \psi$ se réduisent à des expressions de la forme $\sum \varrho_{j}\left|x_{j}\right|^{a}$ et $\sum \sigma_{k}\left|y_{k}\right|^{b}$.

Or il est clair qu'on aura toujours $M_{\alpha \beta}^{\prime} \leqq M_{\alpha \beta}$, ces lettres désignant les bornes respectives de la forme et de l'opération bilinéaires. D'autre part, $(\alpha, \beta)$, $\left(\alpha_{1}, \beta_{1}\right)$ et $\left(\alpha_{2}, \beta_{2}\right)$ étant trois points dudit segment, le premier séparant les deux autres, il viendra par le théorème $I^{\prime}$ que

$$
M_{\alpha_{1} \beta}^{\prime} \leqq\left(M_{\alpha_{1} \beta_{1}}^{\prime}\right)^{\frac{\alpha_{2}-\alpha}{\alpha_{3}-\alpha_{1}}}\left(M_{\alpha_{2} \beta_{3}}^{\prime}\right)^{\alpha-\alpha_{2}-\alpha_{1}}
$$

et alors, à plus forte raison,

$$
M_{\alpha \beta}^{\prime} \leqq\left(M_{\alpha_{1} \beta_{1}}\right)^{\frac{\alpha_{2}-\alpha}{\alpha_{2}-\alpha_{1}}}\left(M_{\alpha_{3} \beta_{2}}\right)^{\frac{\alpha-\alpha_{1}}{\alpha_{2}-\alpha_{1}}}
$$

Cette inégalité ayant lieu pour chaque division d'intervalles, on pourra, par ce qui précède, y remplacer $M_{\alpha \beta}^{\prime}$ par $M_{\alpha \beta}$, et alors le théorème III se trouve démontré.

Le théorème IV résulte par les remarques $d u n^{\circ}$ I 5 de la même inégalité.

18. Avant de passer aux transformations fonctionnelles, nous aurons à poser quelques conventions évidentes et rappeler quelques faits connus.

On dira qu'un ensemble est un ensemble nul par rapport à $\varphi(x)$ ou, plus brièvement, un ensemble nul, si l'on peut l'enfermer dans des systèmes d'intervalles sur' lesquels la croissance totale de $\varphi(x)$ est arbitrairement petite. Une fonction sera dite une fonction nulle par rapport $\grave{a} \varphi(x)$ ou, plus brièvement, une fonction nulle, si elle s'annule sauf peut-être dans un ensemble nul. Deux fonctions qui ne diffèrent que par une fonction nulle ayant des intégrales identiques par rapport à $\varphi(x)$ sur des intervalles quelconques, de telles fonctions seront considérées comme identiques.

Quand $\varphi(x)=x$, c'est-à-dire dans le cas de l'intégrale de Lebesgue, les ensembles nuls sont ceux qu'on appelle en général ensembles de mesure nulle, tandis que les fonctions nulles sont celles qui s'annulent, sauf peut-être dans un tel ensemble.

Dans le cas où $\varphi(x)$ est constante par intervalles, les ensembles nuls sont ceux qui ne contiennent aucun point de discontinuité de $\varphi(x)$, tandis que une fonction arbitraire qui s'annule à tous les points de discontinuité sera une fonction nulle. 
Sur les maxima des formes bilinéaires et sur les fonctionnelles linéaires. $\quad 479$

On posera des conventions analogues en ce qui concerne la fonction $\psi(x)$.

19. On dira que $B$ est une opération fonctionnelle linéaire portant sur la classe $L_{\varphi}^{a}$, si $B$ fait correspondre à toute fonction $f$ de cette classe un nombre déterminé $B(f)$ de sorte que les hypothèses suivantes soient satisfaites:

a) L'opération est distributive, c'est-à-dire que pour des constantes $\lambda_{1}$ et $\lambda_{2}$ quelconques, on a

$$
B\left(\lambda_{1} f_{1}+\lambda_{2} f_{2}\right)=\lambda_{1} B\left(f_{1}\right)+\lambda_{2} B\left(f_{2}\right)
$$

b) Il existe une constante $M$ telle que

$$
|B(f)| \leqq M\left(\int|f|^{a} d \varphi\right)^{\frac{1}{a}}
$$

On sait ${ }^{1}$ qu'il existe alors une fonction génératrice $\omega(x)$, appartenant à la classe complémentaire $L_{\varphi}^{a^{\prime}}\left(\frac{\mathrm{I}}{a}+\frac{\mathrm{I}}{a^{\prime}}=\mathrm{I}\right)$ et déterminée à une fonction nulle près telle que l'on ait

$$
B(f)=\int_{x}^{\lambda} f(x) \omega(x) d \varphi(x)=\int f \omega d \varphi
$$

Si $M$ désigne la borne de $B$, c'est-à-dire le plus petit nombre qui suffit pour que l'inégalité (22) soit remplie, on aura

$$
\left(\int|\omega|^{a^{\prime}} d \varphi\right)^{\frac{1}{a^{\prime}}}=M
$$

Ces faits importants permettront de traduire nos résultats concernant les opérations bilinéaires en de tels concernant les transformations fonctionnelles.

20. Admettons qu'une transformation $T=T(f)$ fasse correspondre aux fonctions d'une certaine classe $L_{\varphi}^{a}$ ou, plus généralement, à un ensemble de fonctions embrassant plusieurs classes $L_{\varphi}^{a}$ certaines fonctions, définies dans le même intervalle que $\psi(x)$, la correspondance étant distributive, c'est-à-dire que pour des constantes arbitraires $\lambda_{1}$ et $\lambda_{2}$ on ait

$$
T\left(\lambda_{1} f_{1}+\lambda_{2} f_{2}\right)=\lambda_{1} T\left(f_{1}\right)+\lambda_{2} T\left(f_{2}\right)
$$

1 Cf. p. ex. Radon, l. c. p. 1371. 
Supposons en outre que les transformées des fonctions de la classe $L_{\varphi}^{a}$ fassent partie d'une certaine classe $L_{\psi}^{c}$ ou qu'elles remplissent cette classe et, de plus, qu'il existe un nombre $M^{*}$ tel qu'on ait toujours

$$
\left(\int|T(f)|^{c} d \psi\right)^{\frac{1}{c}} \leqq M^{*}\left(\int|f|^{a} d \varphi\right)^{\frac{1}{a}}
$$

Alors on dira que $T$ est une transformation fonctionnelle linéaire de $L_{\varphi}^{a}$ en $L_{\psi}^{c}$. Pour chaque couple d'exposants envisagés $a$ et $c$, nous désignerons par $\boldsymbol{M}_{\alpha \gamma}^{*}$, où $\alpha=\frac{\mathrm{I}}{a}$ et $\gamma=\frac{\mathrm{I}}{c}$, le plus petit des nombres $M^{*}$ qui conviennent, ce nombre étant appelé borne de la transformation (par rapport aux exposants envisagés). ${ }^{1}$

Par les conventions posées plus haut et par la nature des choses, deux fonctions de la classe $L_{\varphi}^{a}$ ou de la classe $L_{\psi}^{c}$ qui ne diffèrent que par une fonction nulle par rapport à $\varphi(x)$ ou à $\psi(x)$ respectivement sont considérées comme identiques. Même le cas est admis où ni $f$ ni $T(f)$ ne sont définies qu'à des fonctions nulles près, c'est-à-dire que ces fonctions prennent des valeurs arbitraires, chacune dans un ensemble nul par rapport à $\varphi(x)$ ou à $\psi(x)$ respectivement, Souvent il sera même naturel de ne pas s'occuper des valeurs que $f$ ou $T(f)$ prennent dans un certain ensemble nul. Tel sera le cas p. ex. pour $f$, si la fonction non décroissante $\varphi(x)$ est constante par intervalles. Alors, on ne s'occupera que des valeurs que $f$ prend aux points de discontinuité de cette fonction.

21. Cela posé, considérons une opération bilinéaire $A(f, g)$ portant sur un certain champ $\left(L_{\varphi}^{a}, L_{\psi}^{b}\right)$, avec la borne $M$. En fixant alors la fonction $f(x)$ et en faisant varier $g(x)$, cette opération fera correspondre à toute fonction $g(x)$ de la classe $L_{\psi}^{b}$ un nombre bien déterminé $B(g)$. La correspondance sera distributive, et on aura aussi

$$
|B(g)|=|A(f, g)| \leqq M^{\prime}\left(\int|f|^{a} d \varphi\right)^{\frac{1}{a}}\left(\int|g|^{b} d \psi\right)^{\frac{1}{b}}
$$

$M^{\prime} \leqq M$ désignant le plus petit nombre (dépendant en général de $f$ ) qui convient.

Il s'agira donc d'une opération linéaire pour les fonctions $g(x)$. Alors, par ce qu'on a dit plus haut, il existera une fonction $\omega(x)$, appartenant à la classe

\footnotetext{
1 Pour la signification de $M_{0}^{*}$ et de $M_{\gamma 0}^{*} c f$. la note 2 p. 477 .
} 
$L_{\psi}^{b^{\prime}}$, où $\frac{\mathrm{I}}{b}+\frac{\mathrm{I}}{b^{\prime}}=\mathrm{I}$, bien déterminée à une fonction nulle près et telle que l'on ait

$$
B(g)=\int g \omega d \psi
$$

avec

$$
\left(\int|\omega|^{b^{\prime}} d \psi\right)^{\frac{1}{b^{\prime}}}=M^{\prime}\left(\int|f|^{a} d \varphi\right)^{\frac{1}{a}}
$$

Il en découle que le passage de $f(x)$ à $\omega(x)$ est une transformation fonctionnelle linéaire de $L_{q p}^{a}$ en $L_{\psi}^{b^{\prime}}$, avec la borne $M$. Un raisonnement analogue pour le cas où l'on aura interverti le rôle des fonctions $f(x)$ et $g(x)$ conduira à une transformation de $L_{\psi}^{b}$ en $L_{\varphi}^{a^{\prime}}$, avec la même borne.

C'est-à-dire que toute opération bilinéaire qui porte sur un champ $\left(L_{\phi p}^{a}, L_{\psi}^{b}\right)$ donne lieu à deux transformations linéaires, transposées l'une à l'autre, l'une menant de $L_{\varphi}^{a}$ en $L_{\psi}^{b^{\prime}}$, l'autre de $L_{\psi}^{b}$ en $L_{\varphi}^{a^{\prime}}$, les bornes étant identiques. Inversement, de telles transformations donnent toujours lieu à une opération bilinéaire portant sur ledit champ. Admettons p. ex. que $T$ soit une transformation de $L_{\varphi}^{a}$ en $L_{\psi}^{b^{\prime}}$. Il suffit de poser

$$
A(f, g)=\int g T(f) d \psi
$$

pour obtenir l'opération bilinéaire cherchée.

22. Le lien intime qu'on vient d'établir entre les opérations bilinéaires et les transformations linéaires permet d'énoncer les théorèmes suivants, équivalents aux théorèmes III et IV.

V. Soit $T$ une transformation fonctionnelle linéaire de certaines classes $L_{\Phi}^{a}$ en certaines classes correspondantes $L_{\psi}^{c}$, la correspondance entre a et c étant telle. que l'on ait toujours $c \geqq a$ et que, de plus, le point $(\alpha, \gamma)$, où $\alpha=\frac{\mathrm{I}}{a}$ et $\gamma=\frac{\mathrm{I}}{\mathrm{c}}$, décrive un segment de droite. Alors $\log M_{\alpha \gamma}^{*}$ sera une fonction convexe des points du segment.

VI. Chaque fois qu'on a une transformation fonctionnelle linéaire de $L_{\varphi}^{a_{1}}$ en $L_{\psi}^{c_{1}}$ et de $L_{\varphi}^{a_{2}}$ en $L_{\psi}^{c_{3}}$, avec $c_{1} \geqq a_{1}$ et $c_{2} \geqq a_{2}$, la transformation pourra être étendue $\grave{a}$ tous les couples d'exposants correspondant aux points $(\alpha, \gamma)$.du segment joignant les points $\left(\alpha_{1}, \gamma_{1}\right)$ et $\left(\alpha_{2}, \gamma_{2}\right)$.

61-2661. Acta mathematica. 49. Imprimé le 11 janvier 1927. 


\section{Applications.}

23. Soit $f(x)$ une fonction réelle ou non, définie dans l'intervalle $o \leqq x \leqq \mathrm{I}$, intégrable au sens de Lebesgue et soient

$$
C_{k}=\int_{0}^{1} f(x) e^{-2 k \pi i x} d x \quad(k=0, \pm \mathrm{I}, \pm 2, \ldots)
$$

ses constantes de Fourier. ${ }^{1}$

Posons

$$
J_{a}=\left(\int_{0}^{1}|f(x)|^{a} d x\right)^{\frac{1}{a}} ; \quad S_{a}=\left(\sum_{k=-\infty}^{+\infty}\left|C_{k}\right|^{a}\right)^{\frac{1}{a}}
$$

Soient de plus $a>\mathrm{I}$ et $c>\mathrm{I}$ deux exposants liés par la relation $\frac{\mathrm{I}}{a}+\frac{\mathrm{I}}{c}=\mathrm{I}$.

On aura les théorèmes suivants.

A. Si $a \leqq c$, et si la série $\sum\left|C_{k}\right|^{a}$ est convergente, les $C_{k}$ sont les constantes de Fourier d'une fonction $f$, intégrable ainsi que $|f|^{c}$, et il est $J_{c} \leqq S_{a}$.

B. Lorsque $a \leqq c$ et que $f$ est intégrable ainsi que $|f|^{a}$, la série $\sum\left|C_{k}\right|^{c}$ sera convergente et $S_{e} \leqq J_{a}$.

Ces théorèmes sont dus à MM. W. H. Young et F. HAdsdorfF. ${ }^{2}$ M. Young ayant d'abord établi le cas particulier où $c$ est un entier pair, M. HAUSDoRfF a démontré les théorèmes pour le cas général.

M. F. RIEsz ${ }^{3}$ a montré que les théorèmes ci-dessus subsistent lorsqu'on introduit au lieu des fonctions $e^{2 k \pi i x}$, un système quelconque de fonctions orthogonales et normées dont le module ne dépasse pas l'unité, définies d'ailleurs sur un intervalle quelconque. Plus généralement, si les fonctions du système ne dépassent pas en module le nombre $\mathfrak{M}$, les inégalités ci-dessus seront à remplacer par.

$$
J_{c} \leqq \mathfrak{M}^{\frac{2-a}{a}} S_{a} \quad \text { et } \quad S_{c} \leqq \mathfrak{M}^{\frac{2-a}{a}} J_{a}
$$

respectivement.

${ }^{1}$ Ce n'est que pour simplifier les énoncés que nous considérons ici les fonctions $e^{2 k \pi i x}$ au lieu des fonctions $e^{k i x}$.

2 l. c. note 2 , p. 466 .

l. c. note 3 , p. 466 . 
Sur les maxima des formes bilinéaires et sur les fonctionnelles linéaires. 483

24. On pourrait déduire ces théorèmes comme cas particuliers de deux autres théorèmes résultant, par passage à la limite, du théorème II et que le lecteur pourra formuler sans peine.

Il s'y agit de la convexité pour $o \leqq \gamma \leqq \alpha \leqq \mathrm{I}$, du logarithme de la borne supérieure du rapport

$$
\left(\int_{A}^{B}\left|\sum C_{j} \tau_{j}(x)\right|^{\frac{1}{\gamma}} d x\right)^{\gamma}:\left(\sum\left|C_{j}\right|^{\frac{1}{\alpha}}\right)^{\alpha}
$$

et de celle du rapport

$$
\left(\Sigma\left|\int_{A}^{B} f(x) \tau_{j}(x) d x\right|^{\frac{1}{\gamma}}\right)^{\gamma}:\left(\int_{A}^{B}|f(x)|^{\frac{1}{\alpha}} d x\right)^{\alpha}
$$

les $C_{j}$ étant variés dans le premier cas et $f(x)$ dans le second. Les $\tau_{j}$ sont des fonctions données arbitraires, orthogonales ou non.

Nous préférons montrer comment les théorèmes précités rentrent dans l'ordre d'idées de nos résultats concernant les transformations fonctionnelles.

25. Nous établirons à titre d'exemple la seconde des inégalités (25).

Considérons un système de fonctions orthogonales et normées $\omega_{k}(x)$ définies dans l'intervalle $A \leqq x \leqq B$ et telles que l'on ait $\left|\omega_{k}(x)\right| \leqq M$. Soit de plus $\varphi(x)=x$ pour $A \leqq x \leqq B$ et soit $\psi(x)$, dans l'intervalle $(0, \infty)^{1}$, égale au plus grand nombre entier compris dans $x$. La transformée $T(f)$ de $f$ sera, par définition, égale à

$$
C_{k}=\int_{A}^{B} f(t) \overline{\omega_{k}(t)} d t
$$

pour les valeurs entières $k$ de $x$, tandis qu'elle sera arbitraire pour $x$ non entier.

On aura

${ }^{1}$ Dans le cas du théorème de Young-Hausdorff, on définira $\psi(x)$ d'une manière analogue dans l'intervalle $(-\infty,+\infty)$. Ajoutons qu'on pourrait éviter les intégrales de Stieltjes-Lebesgue et se contenter d'intégrales ordinaires par le procédé un peu artificiel de poser, dans le cas du texte p. ex., $\psi(x)=x$, pour $o \leqq x \leqq \infty$ et $T(f)=C_{k}$ pour $k \leqq x<k+\mathrm{I}$.

$2 \overline{\omega_{k}(t)}$ désigne la valeur conjuguée de $\omega_{k}(t)$. 


$$
\int|T(f)|^{\frac{1}{\gamma}} d \psi=\sum_{k=0}^{\infty}\left|C_{k}\right|^{\frac{1}{\gamma}}
$$

de plus $M_{1,0}^{*} \leqq \mathfrak{M}$ et $M_{\frac{1}{2}, \frac{1}{2}}^{*} \leqq \mathrm{I}$. En effet, la première inégalité résulte de ce que $M_{1,0}^{*}$ signifie la borne supérieure des $\left|C_{k}\right|, f$ étant variée sous la condition $\int_{A}^{B}|f(x)| d x \leqq \mathrm{I},{ }^{1}$ tandis que la seconde n'est que l'inégalité bien connue de Bessel.

Le segment limité par les points $(\mathrm{I}, \mathrm{O})$ et $\left(\frac{1}{2}, \frac{1}{2}\right)$ ayant pour équation $\gamma=\mathrm{I}-\alpha$, où $\frac{1}{2}<\alpha=\frac{\mathrm{I}}{a}<\mathrm{I}$, il résulte du théorème VI que, chaque fois que $f$ et $|f|^{a}$ sont intégrables, la série $\sum\left|C_{k}\right|^{c}$, où $e=\frac{\mathrm{I}}{\gamma}=\frac{\mathrm{I}}{\mathrm{I}-\alpha}$, sera convergente. L'inégalité à démontrer résulte alors de la convexité de $\log M_{\alpha, 1-\alpha}^{*}$, assurée par le théorème $\nabla$. On a en effet, tout comme au $n^{\circ}$ I,

$$
M_{\alpha, 1-\alpha}^{*} \leqq\left(M_{i, 0}^{*}\right)^{\frac{\alpha-\frac{1}{2}}{1-\frac{1}{2}}}\left(M_{\frac{1}{2}, \frac{1}{2}}^{*}\right)^{\frac{1-\alpha}{1-\frac{1}{2}}} \leqq M^{2 \alpha-1}=\mathfrak{M}^{\frac{2-a}{a}}
$$

La première des inégalités (25) résulte par un raisonnement analogue ou encore par le fait général établi plus haut savoir que deux transformations transposées admettent la même borne, égale à celle de l'opération bilinéaire correspondante.

26. L'analogue des théorèmes de Young-Hausdorff pour l'intégrale de Fourier fut démontré par M. Tітснмarsh. ${ }^{2}$ Les raisonnements qui précèdent s'appliquent sans difficulté à ce cas-ci ${ }^{3}$ et à d'autres intégrales analogues; nous avons l'intention d'y revenir à une autre occasion.

27. Passons maintenant à une autre application, concernant quelques théorèmes que j'ai établis ailleurs ${ }^{4}$ au sujet des fonctions conjuguées. ${ }^{5}$

${ }^{1}$ Cf. la note 2 p. 477 et la note I p. 480.

2 E. C. TITchmaRsh, A contribution to the theory of Fourier transforms, Proc. London Math. Soc. (2), t. 23 (1924), p. 279-287.

3 Au lieu de l'inégalité de Bessel on s'appuie sur un théorème connu de M. Plancherel.

4 M. Rresz, I) Les fonctions conjuguées et les séries de Fourier, Comptes rendus, t. 178 (28 avril I924) p. I464;2) Sur les fonctions conjuguées, va paraître dans la Math. Zeitschr.

5 Cf. aussi E. C. TitchmaRsh, Reciprocal formulæ involving series and integrals, Math. Zeitschr. t. 25 (1926) p. $32 \mathrm{I}-347$. 
Sur les maxima des formes bilinéaires et sur les fonctionnelles linéaires.

485

Désignons par $f(x)$ une fonction intégrable au sens de M. Lebesgue dans l'intervalle $(0,2 \pi)$ et écrivons l'expression

$$
\bar{f}(x)=\frac{\mathrm{I}}{2 \pi} \int_{0}^{2 \pi} f(t) \cot \frac{t-x}{2} d t
$$

Complétant certains résultats de M. Fatou, M. Plessner a démontré que l'intégrale du second membre existe presque partout, si l'on la prend au sens de la valeur principale de CAUchx. ${ }^{1}$

En désignant par $L^{a}$, où $a>\mathrm{I}$, la classe des fonctions $f(x)$ intégrables avec $|f|^{a}$, on a les théorèmes que voici.

A. Si la fonction périodique $f(x)$, de période $2 \pi$, appartient à la classe $L^{a}$, la fonction $\vec{f}(x)$ y appartient également, et l'on a

$$
\left(\int_{0}^{2 \pi}|\bar{f}(x)|^{a} d x\right)^{\frac{1}{a}} \leqq M_{a}\left(\int_{0}^{2 \pi}|f(x)|^{a} d x\right)^{\frac{1}{a}}
$$

B. Soit $f(x)$ une fonction de la classe $L^{a}$ par rapport à l'intervalle $(-\infty,+\infty)$ et formons l'intégrale

$$
\bar{f}(x)=\frac{\mathrm{I}}{\pi} \int_{-\infty}^{+\infty} \frac{f(t)}{t-x} d t
$$

dont la valeur principale exiște presque partout. On a alors une inégalité analogue à (28), le facteur constant étant le même.

C. Pour deux fonctions $f(x)$ et $g(x)$ appartenant à des classes complémentaires $L^{a}$ et $L^{a^{\prime}}$, où $\frac{\mathrm{I}}{a}+\frac{\mathrm{I}}{a^{\prime}}=\mathrm{I}$, on a, sous les hypothèses du premier théorème,

(30) $\frac{\mathrm{I}}{2 \pi}\left|\int_{0}^{2 \pi} \int_{0}^{2 \pi} f(x) g(y) \cot \frac{x-y}{2} d x d y\right| \leqq M_{a}\left(\left.\int_{0}^{2 \pi}|f(x)|\right|^{a} d x\right)^{\frac{1}{a}}\left(\int_{0}^{2 \pi}|g(x)|^{a^{\prime}} d x\right)^{\frac{1}{a^{\prime}}}$ et sous celles du second

${ }^{1}$ A. Puessner, Zur Theorie der konjugierten trigonometrischen Reihen, Inauguraldissertation, Giessen, 1923. 


$$
\frac{\mathrm{I}}{\pi}\left|\int_{-\infty}^{+\infty} \int_{-\infty}^{+\infty} \frac{f(x) g(y)}{x-y} d x d y\right| \leqq M_{a}\left(\int_{-\infty}^{+\infty}|f(x)|^{a} d x\right)^{\frac{1}{a}}\left(\int_{-\infty}^{+\infty}|g(x)|^{a^{\prime}} d x\right)^{\frac{1}{a^{\prime}}}
$$

les intégrales doubles existant toujours, si l'on les prend au sens de la valeur principale de $\mathrm{C}_{\mathrm{AUCH}}$, c'est-à-dire comme limite des intégrales étendues aux valeurs $|x-y| \geqq \varepsilon>0$.

Bien entendu, nous avons désigné par $M_{a}$ la plus petite constante convenable.

28. Les intégrales doubles figurant en (30) et (3I) définissent évidemment des opérations bilinéaires pour les classes complémentaires $L^{a}$ et $L^{a^{\prime}}$. La valeur de ces intégrales restant la même, au signe près, si l'on intervertit le rôle des fonctions $f$ et $g$, l'opération est symétrique gauche. Il s'ensuit que $M_{a^{\prime}}=M_{a}$. On a de plus $M_{2}=$ I et l'on démontre facilement que $M_{a}>$ I pour toute autre valeur de $a$ et que $M_{a}$ devient infini lorsque $a$ tend vers I ou vers l'infini: D'autre part, il résulte de nos théorèmes III ou $V$ que $\log M_{a}$ est une fonction convexe de $\alpha=\frac{\mathrm{I}}{a}$. En résumé, $\log M_{a}$ et alors $M_{a}$ sont des fonctions convexes de $\alpha=\frac{\mathrm{I}}{a}$ pour $\mathrm{o} \leqq \alpha \leqq \mathrm{I}$, symétriques par rapport à $\alpha=\frac{1}{2}$, et vont en croissant vers l'infini quand $\alpha$ va de $\frac{1}{2}$ à o ou à $\mathrm{I}$.

Quant à l'ordre de croissance de $M_{a}$, il ressort des recherches de $M$. Titchmarsh qu'il existe deux constantes $H$ et $K$ telles que pour $a \geqq 2$,

$$
H a \leqq M_{a} \leqq K a^{\frac{4}{3}}{ }^{1}
$$

Or, dans le second de mes travaux cités, j'ai démontré que pour $a$ entier et pair, on a $M_{a}<a: \log 2$. Par la croissance de $M_{a}$ pour $a>2$, il en résulte l'existence d'une constante $K$ telle que l'on a pour tout exposant $a \geqq 2, M_{a} \leqq K a$. La combinaison de notre résultat avec celui de M. Titchmarsh donne en définitive que pour $a \geqq 2$,

$$
H a \leqq M_{a} \leqq K a
$$

29. Voyons maintenant quel parti on peut tirer des résultats généraux du travail présent pour la démonstration des théorèmes $d u n^{\circ} 27$. Nous nous contentons de quelques indications sommaires. Considérons p. ex. le théorème $A$. Pour $a=2$, la démonstration de ce théorème est immédiate. On l'obtient p. ex. 
Sur les maxima des formes bilinéaires et sur les fonctionnelles linéaires. $\quad 487$ par le théorème de Parseval en observant que les séries de Fourier de $f(x)$ et de $\bar{f}(x)$ sont conjuguées.

La démonstration n'étant immédiate que pour l'exposant 2 , on ne pourra imiter le procédé suivi dans les applications précédentes, où l'on disposait de deux exposants distingués. Voici tout de même comment on peut ramener le cas d'un exposant arbitraire à celui, beaucoup plus facile, des entiers pairs.

Admettons que le théorème $\mathrm{A}$ soit démontré pour l'entier pair $a=2 n$. Alors, par la symétrie gauche, le théorème se trouvera en même temps démontré pour $a^{\prime}=2 n:(2 n-\mathrm{I})$ et enfin, par le théorème $\nabla I$, pour tout exposant situé entre $a$ et $a^{\prime}$.

30. Comme dernière application considérons le problème suivant.

Soit

$$
f(x) \sim \sum_{k=-\infty}^{+\infty} C_{k} e^{k i x}
$$

une série de Fourier, c'est-à-dire que les $C_{k}$, les constantes de Fourier de $f(x)$, se déterminent par les relations

$$
C_{k}=\frac{\mathrm{I}}{2 \pi} \int_{0}^{2 \pi} f(x) e^{-k i x} d x
$$

Considérons une suite de nombres $\lambda_{k}$ telle que, chaque fois que les $C_{k}$ sont les constantes de Fourier d'une fonction de la classe $L^{a}$, les produits $\lambda_{k} C_{k}$ soient les constantes de Fourier d'une fonction de la classe $L^{c}$. Cette fonction sera désignée par $T(f)$ et appelée la transformée de $f(x)$, tandis que la suite sera dite une suite de facteurs du type $(a, c)$.

Il est très facile d'établir, par un raisonnement connu remontant à $M$. Lebesgoe et à $M$. HaAR, qu'il existe un nombre $M$, attaché à la suite, tel qu'on ait

$$
\left(\int_{0}^{2 \pi}|T(f)|^{c} d x\right)^{\frac{1}{c}} \leqq M\left(\int_{0}^{2 \pi}|f(x)|^{a} d x\right)^{\frac{1}{a}}
$$

Ici encore, c'est évidemment le plus petit nombre $M$ convenable qui intéresse et qui est la borne de la transformation.

31. Les suites du type $(2,2)$ ne sont que les suites bornées; cela ressort immédiatement du théorème de Riesz-Fischer. 
Les suites du type (I, I) sont caractérisées par le fait que la série

$$
\sum_{k=-\infty}^{+\infty} \lambda_{k} \frac{e^{k i x}}{k i}
$$

où l'accent indique la suppression de l'indice zéro, est la série de Fourier d'une fonction à variation bornée $G(x)$.

Ces suites $\left(\lambda_{k}\right)$ sont aussi identiques à celles qui transforment les fonctions bornées en des fonctions bornées, ${ }^{1}$ c'est-à-dire que ces suites sont en même temps celles du type $(\infty, \infty)$, les fonctions bornées correspondant d'une marière connue au cas limite $a=\infty$.

Ajoutons que, toutes les fois que la fonction $G(x)$ donnée par la série (33) est à variation bornée, la transformation peut être mise sous la forme d'une intégrale de Stieltjes-Lebesgue

$$
T[f(x)]=\frac{\mathrm{I}}{2 \pi} \int_{0}^{2 \pi} f(x-t) d\left[G(t)+\lambda_{0} t\right] .
$$

On sait assez peu des suites du type $(a, a)$ pour d'autres valeurs que $a=\mathrm{I}, 2, \infty$. On démontre sans peine - nous y reviendrons tout à l'heure que, tout comme les suites des types $(I, I)$ et $(\infty, \infty)$ sont identiques, il en est de même des types $(a, a)$ et $\left(a^{\prime}, a^{\prime}\right)$, où, comme toujours, $\frac{1}{a}+\frac{1}{a^{\prime}}==\mathbf{I}$. De plus, il est très facile de montrer que toute suite du type $(a, a)$ est bornée, e'est-à-dire qu'elle est en même temps du type $(2,2)$. Pour le voir, il suffit d'appliquer l'inégalité (32) aux fonctions $e^{k i x}$. On démontre encore assez facilement que toute suite du type $(\mathrm{I}, \mathrm{I})$ ou $(\infty, \infty)$ est en même temps du type $(a, a)$ pour $a$ quelconque. Ce fait est un cas particulier du théorème qui suit; d'ailleurs il résulte aussi d'une manière directe en appliquant l'inégalité de Hölder à la formule (34).

32. Les faits qu'on vient d'indiquer suggèrent le théorème suivant.

1 Les résultats concernant les suites des types $(I, I)$ ou $(\infty, \infty)$ que nous avons indiqués sont dus à MM. W. H. Young et S. SzIDon; W. H. Young, On Fourier series and functions of bounded variation, Lond. Roy. Soc. Proc. t. 88 (1913), p. 56I-568, On a condition that a trigonometrical series should have a certain form, ibid. p. 569-574; S. Szrdon, Reihentheoretische Sätze und ihre Anwendungen in der Theorie der Fourierschen Reihen, Math. Zeitschr. t. IO (1921), p. I21-I27. M. Fekete a montré (Über Faktorenfolgen welche die "Klasse" einer Fourierschen Reihe unverändert lassen, Szeged Acta Univ. Franc.-Jos. t. I (1923), p. I48-166) que lesdites suites sont aussi les suites de facteurs qui transforment bien d'autres classes de fonctions (fonetions continues, fonctions intégrables au sens de Riemann etc.) en elles-mêmes. 
Sur les maxima des formes bilinéaires et sur les fonctionnelles linéaires. $\quad 489$

Toute suite du type $\left(a_{0}, a_{0}\right)$ est en même temps une suite de type (a, a) pour tout nombre a situé entre $a_{0}$ et $a_{0}^{\prime}$, les bornes correspondant aux exposants intermédiaires ne dépassant pas la borne commune correspondant aux extrémités.

Ce théorème sera une conséquence immédiate des théorèmes VI et $\mathrm{V}$, dès qu'on aura démontré que les suites des types $(a, a)$ et $\left(a^{\prime}, a^{\prime}\right)$ sont identiques et que les bornes correspondant à une suite particulière sont les mêmes, cette suite étant considérée comme une suite du premier ou du second type.

Or ceci ressort du théorème suivant qui est de caractère symétrique et qui, de son côté, découle assez facilement de ce que j'ai démontré ailleurs au sujet du théorème de Parseval. ${ }^{1}$

Pour que la suite $\left(\lambda_{k}\right)$ soit une suite du type $(a, a)$ ou $\left(a^{\prime}, a^{\prime}\right)$, il faut et il suffit que la série $\sum_{k=-\infty}^{+\infty} \lambda_{k} C_{k} D_{k}$ soit convergente, les $C_{k}$ et les $D_{k}$ désignant les constantes de Fourier de fonctions arbitraires $f(x)$ et $g(x)$ des classes respectives $L^{n}$ et $L^{a^{\prime}}$. Le plus petit nombre $M$ tel qu'on ait

$$
\left|\sum_{k=-\infty}^{+\infty} \lambda_{k} C_{k} D_{k}\right| \leqq \frac{M}{2 \pi}\left(\int_{0}^{2 \pi}|f(x)|^{a} d x\right)^{\frac{1}{a}}\left(\int_{0}^{2 \pi}|g(x)|^{a^{\prime}} d x\right)^{\frac{1}{a^{\prime}}}
$$

est la borne commune, la suite étant envisagée comme suite des types $(a, a)$ ou $\left(a^{\prime}, a^{\prime}\right)$.

On pourrait aussi s'appuyer sur le fait plus élémentaire qui suit et qui ne fait intervenir que des sommes finies.

Pour que la suite $\left(\lambda_{k}\right)$ soit du type $(a, a)$ ou du type $\left(a^{\prime}, a^{\prime}\right)$, avec la borne $M$, il faut et il suffit que (35) ait lieu pour toutes les fonctions $f(x)$ et $g(x)$ qui sont des combinaisons linéaires à un nombre fini de termes des fontions $e^{k i x}$, c'està-dire des polynomes trigonométriques, $M$ étant le plus petit nombre qui y convient.

La nécessité de la condition est évidente. D'autre part, on voit facilement que la condition est suffisante; on n'aura qu'à observer que lesdits polynomes sont partout denses dans les classes respectives au sens $d u n^{\circ}{ }_{1} 5$.

Voici encore une généralisation qui se démontre par un raisonnement analogue.

1 Comptes rendus, l. c., Math. Zeitschr. l. c.

62-2661 Acta mathematica. 49. Imprimé le 12 janvier 1927. 
Considérons des suites du type $(a, c)$, avec $c \geqq a .^{1}$ Posons, comme plus haut, $\alpha=\frac{\mathrm{I}}{a}, \gamma=\frac{\mathrm{I}}{e}$.

Cela posé, toute suite du type $\left(a_{0}, c_{0}\right)$ est aussi du type $(a, c)$ pour tout point $(\alpha, \gamma)$ appartenant au segment de droite limité par les points $\left(\alpha_{0}, \gamma_{0}\right)$ et $\left(\alpha_{0}{ }^{\prime}, \gamma_{0}{ }^{\prime}\right)$, les bornes correspondant aux points intermédiaires ne dépassant pas la borne commune qui correspond aux extrémités.

33. Considérons enfin la suite de facteurs particulière $\lambda_{0}=0, \lambda_{k}= \pm i$ pour $k \lessgtr o$ qui transforme toute série trigonométrique

$$
\sum_{k=-\infty}^{+\infty} C_{k} e^{k i x}
$$

dans sa conjuguée

$$
\sum_{k=-\infty}^{-1} i C_{k} e^{k i x}-\sum_{k=1}^{+\infty} i C_{k} e^{k i x}
$$

En particulier, la première série étant la série de Fourier d'une fonction $f(x)$ de la classe $L^{a}$, la seconde sera celle de la fontion $\bar{f}(x)$, donnée par la formule (27) qui, par le théorème $\mathrm{A}$ du $\mathrm{n}^{\circ} 27$, appartient à la même classe. Le problème des fonctions conjuguées rentre donc dans l'ordre d'idées des suites de facteurs. D'autre part, on voit que ladite suite particulière, et alors celle où l'on aura remplacé $\pm i$ par $\pm \mathrm{I}$, est, pour tout $a$ fini $>\mathrm{r}$, une suite de facteurs du type $(a, a)$ avec la borne $M_{a}$. Toute translation d'indices fournissant une suite analogue, on en déduit par soustraction les suites..., $0,0, \frac{1}{2}, \mathrm{I}, \mathrm{I}, \ldots$, I, I, $\frac{1}{2}, \mathrm{o}, \mathrm{o}, \ldots$, les bornes de ces suites, considérées eomme suites de facteurs du type $(a, a)$, étant encore $\leqq M_{a}$. Il s'ensuit que les bornes des suites $\ldots, \mathrm{o}, \mathrm{o}, \mathrm{I}, \mathrm{I}, \ldots, \mathrm{I}, \mathrm{I}, \mathrm{o}, \mathrm{o}, \ldots$ sont $\leqq M_{a}+\mathrm{I}$. Par ces dernières suites, on passe de la fonction $f(x)$ aux sommes partielles de sa série de Fourier et l'on trouve en particulier

$$
\int_{0}^{2 \pi}\left|s_{m, n}(x)\right|^{a} d x=\int_{0}^{2 \pi}\left|\sum_{k=m}^{n} C_{k} e^{k i x}\right|^{a} d x \leqq\left(M_{a}+\mathrm{I}\right)^{a} \int_{0}^{2 \pi}|f(x)|^{a} d x,
$$

résultat que j'ai déjà donné ailleurs sous une forme moins précise.

1 Pour de telles suites $c f$. G. H. HARDy and J. E. Littlewood, Some properties of fractional integrals, Proc. London Math. Soc. t. 23 (I924), Records, p. XXXVII-XLI. 
Sur les maxima des formes bilinéaires et sur les fonctionnelles linéaires. 491

Voici une autre conséqence des remarques qui précèdent qu'on obtient en appliquant la transformation: d'Abel; c'est que toute suite monotone bornée ou, plus généralement, toute suite $\left(\lambda_{k}\right)$ à variation bornée, c'est-à-dire telle que la série $V=\Sigma\left|\lambda_{k+1}-\lambda_{k}\right|$ converge, est encore pour tout $a$ fini $>_{\text {I }}$ une suite du type $(a, a)$.

\section{Questions de réalité.}

34. Retournons maintenant aux formes bilinéaires et substitutions linéaires à un nombre fini de variables et supposons que les coefficients $a_{j k}$ qui y interviennent soient réels. Cela étant, nous allons établir que, tant qu'on se trouve dans le triangle

$$
\alpha \leqq \mathrm{I}, \beta \leqq \mathrm{I}, \alpha+\beta>\mathrm{I},
$$

les systèmes $\left(x_{j}\right)$ et $\left(y_{k}\right)$ qui rendent $|A(x, y)|$ maximum, sous les conditions (I), sont réels, chacun à un facteur arbitraire $e^{i \Theta}$ près.

Il sera un peu plus commode d'établir le fait équivalent, savoir que les systèmes $\left(x_{j}\right)$ qui rendent

$$
\left(\sum_{k=1}^{n}\left|X_{k}\right|^{c}\right)^{\frac{1}{c}}:\left(\sum_{j=1}^{m}\left|x_{j}\right|^{a}\right)^{\frac{1}{a}}
$$

maximum sont, pour $e>a \geqq \mathrm{I}$, réels à un facteur arbitraire près.

Or ceci résulte immédiatement du lemme suivant.

Soient $x_{j}^{\prime}, x_{j}^{\prime \prime}, X_{k}{ }^{\prime}, X_{k}^{\prime \prime}(j=\mathrm{I}, 2, \ldots, m ; k=\mathrm{I}, 2, \ldots, n)$ des nombres réels et soit $\zeta=\xi+i \eta$ une variable complexe. Admettons en outre que les deux systèmes $\left(x_{j}^{\prime}\right)$ et $\left(x_{j}^{\prime \prime}\right)$ ne soit pas proportionnels l'un à l'autre. Soit de plus $c>a \geqq \mathrm{I}$. Alors en posant

$$
\varphi(\zeta)=\left(\sum_{k=1}^{n}\left|X_{k}^{\prime}+\zeta X_{k}^{\prime \prime}\right|^{c}\right)^{\frac{1}{c}}:\left(\sum_{j=1}^{m}\left|x_{j}^{\prime}+\zeta x_{j}^{\prime \prime}\right|^{a}\right)^{\frac{1}{a}},
$$

$\varphi(\zeta)$ atteint son maximum sur l'axe réel, l'infini y-compris.

Admettons en effet le contraire, c'est-à-dire que $\varphi(\zeta)$ atteigne son maximum pour une valeur finie $\zeta=\xi+i \eta$ et telle que $\eta \neq 0$. Alors on aura 


$$
\frac{\partial \varphi}{\partial \xi}=\mathrm{o}, \frac{\partial \varphi}{\partial \eta}=\mathrm{o}, \text { ou }\left(\frac{\partial}{\partial \xi}-i \frac{\partial}{\partial \eta}\right) \varphi=0
$$

Or on en déduit facilement en utilisant l'inégalité de Hölder et l'hypothèse $c>a$, que l'on a au même point

$$
\frac{\partial^{2} \varphi}{\partial \xi^{2}}+\frac{\partial^{2} \varphi}{\partial \eta^{2}}=\left(\frac{\partial}{\partial \xi}+i \frac{\partial}{\partial \eta}\right)\left(\begin{array}{c}
\partial \\
\partial \xi
\end{array}-i \frac{\partial}{\partial \eta}\right) \varphi>0 .
$$

Par conséquent, il ne pourra s'agir de maximum dans le point en question.

Le lemme établi, notre énoncé en ressort, en décomposant les variables $x_{j}=x_{j}^{\prime}+i x_{j}^{\prime \prime}$ et les formes $X_{k}=X_{k}{ }^{\prime}+i X_{k}^{\prime \prime}$ en parties réelle et imaginaire et en supposant par impossible $\zeta=i$.

35. Quant aux points $d u$ triangle situés sur le côté $\alpha+\beta=\mathrm{I}$, il résulte par continuité qu'il existe toujours des systèmes réels des variables fournissant le maximum. Mais il peut arriver que la même valeur maximée sera aussi atteinte pour des systèmes qui ne sont pas proportionnels à des systèmes réels. Il suffit de montrer que, pour $c=a$, le fait analogue pourra arriver pour l'expression (37). Or il en sera ainsi dans l'exemple évident

$$
X_{j}=x_{j} \quad(j=\mathrm{I}, 2, \ldots, m) .
$$

Pour $e=a$, le rapport étant constant, il atteint son maximum pour n'importe quel système, reeel ou non.

Il sera utile de considérer le même exemple pour $c>a$ et $c<a$.

Dans le premier cas, les systèmes fournissant le maximum sont ceux où toutes les variables s'annulent, sauf une d'entre elles. De tels systèmes sont réels à un facteur arbitraire près, ce qui est bien d'accord avec le résultat établi au numéro précédent.

Pour $c<a$, l'inégalité de Hölder ou l'inégalité (4') donnent

$$
\left(\sum_{j=1}^{m}\left|x_{j}\right|^{c}\right)^{\frac{1}{c}} \leqq m^{\frac{1}{c}-\frac{1}{a}}\left(\sum_{j=1}^{m}\left|x_{j}\right|^{a}\right)^{\frac{1}{a}}
$$

le signe d'égalité n'ayant lieu que quand tous les $\left|x_{j}\right|$ sont égaux. Tout tel système, réel ou non, fournira alors, dans l'exemple particulier qu'on considère, le maximum de (37).

En retournant à la forme bilinéaire, on voit que tant que le point $(\alpha, \beta)$ se trouve dans le triangle 
Sur les maxima des formes bilinéaires et sur les fonctionnelles linéaires.

$$
0 \leqq \alpha, 0 \leqq \beta, \alpha+\beta \leqq \mathrm{I}
$$

les systèmes qui fournissent le maximum pourront être réels ou non.

36. En supprimant maintenant l'hypothénuse du triangle en question, on aura le triangle

$$
\mathrm{O} \leqq \alpha, 0 \leqq \beta, \alpha+\beta<\mathrm{I}
$$

Nous allons montrer que pour chaque point de ce dernier triangle, on pourra construire des exemples où le maximum n'est fourni que par des systèmes non réels. En comparant ce fait avec le résultat établi au $n^{\circ} 34$, on voit qu'il y a une différence essentielle entre les deux triangles (36) et (40), le côté commun possédant dans un certain sens le caractère de tous les deux.

Pour construire les exemples en question, il suffira encore de considérer l'expression (37) pour $\mathrm{I} \leqq c<a$.

En posant ${ }^{1} m=n=3$ et en modifiant un peu l'exemple précédent, considérons la substitution

$$
X_{j}=x_{j}-\varepsilon\left(x_{1}+x_{2}+x_{3}\right) \quad(j=1,2,3) .
$$

$\varepsilon$ étant un nombre positif très petit. Montrons que, étant donné un couple de valeurs $(a, c)$ telles que $\mathrm{I} \leqq c<a$, on pourra choisir $\varepsilon$ assez petit pour que le maximum de

$$
\left(\left|X_{1}\right|^{c}+\left|X_{2}\right|^{c}+\left|X_{3}\right|^{c}\right)^{\frac{1}{c}}:\left(\left|x_{1}\right|^{a}+\left|x_{2}\right|^{a}+\left|x_{3}\right|^{a}\right)^{\frac{1}{a}}
$$

ne soit atteint pour aucun système réel.

Remarquons d'abord que le rapport (42) a évidemment la valeur $3^{\frac{1}{c}-\frac{1}{a}}$ pour tout système tel que $\left|x_{1}\right|=\left|x_{2}\right|=\left|x_{3}\right|$ et que $x_{1}+x_{2}+x_{3}=0$, p. ex. pour le système $x_{1}=\mathrm{I}, x_{2}=e^{\frac{2 \pi i}{3}}, x_{3}=e^{-\frac{2 \pi i}{3}} ;$ chaque système qui remplit les conditions précédentes étant d'ailleurs, à l'ordre près, proportionnel au système particulier indiqué.

Pour voir que le maximum n'est atteint pour aucun système réel, il suffit de montrer que les valeurs que le rapport (42) admet pour les valeurs réelles des variables, sont inférieures à $3^{\frac{1}{c}-\frac{1}{a}}$.

\footnotetext{
${ }^{1}$ Le raisonnement qui suit tient pour $m$ impair quelconque.
} 
On peut admettre, sans restreindre la généralité, que $\left|x_{1}\right|^{2}+\left|x_{9}\right|^{n}+\left|x_{s}\right|^{n}=3$. En fixant alors un voisinage très étroit des systèmes $(1, I, I)$ et $(I, I,-I)$, il est clair, d'après la remarque ajoutée à l'inégalité (38), qu'on pourra déterminer $\varepsilon$ assez petit pour que tout système qui à un facteur commun ou à l'ordre près, ne se trouve dans ledit voisinage fournisse pour le rapport (42) une valeur inférieure à $3^{\frac{1}{c}-{ }^{1}}$. Quant aux systèmes qui se trouvent auxdits voisinages, on aura, suivant le cas,

$\left|X_{1}\right|^{c}+\left|X_{2}\right|^{c}+\left|X_{3}\right|^{c}=\left|x_{1}\right|^{c}+\left|x_{2}\right|^{c}+\left|x_{3}\right|^{c}-c \varepsilon\left(\left|x_{1}\right|^{c-1}+\left|x_{2}\right|^{c-1} \pm\left|x_{3}\right|^{c-1}\right)\left(x_{1}+x_{2}+x_{3}\right)+O\left(\varepsilon^{2}\right)$.

On a d'abord, d'après $(38),\left|x_{1}\right|^{c}+\left|x_{2}\right|^{c}+\left|x_{3}\right|^{i} \leqq 3$. D'autre part, la valeur approchée du terme négatif sera, suivant le cas, $-9 c \varepsilon$ ou $-c \varepsilon$.

On aura donc dans tous les cas, les voisinages en question étant assez petits,

$$
\left(\left|X_{1}\right|^{c}+\left|X_{2}\right|^{c}+\left|X_{3}\right|^{c}\right)^{\frac{1}{c}}<3^{\frac{1}{c}}-\frac{\varepsilon}{2}
$$

c'est-à-dire que le rapport (42) est inférieur à $3^{\frac{1}{c}-\frac{1}{a}}$.

\section{Remarques additionnelles concernant la convexité.}

37. Il reste à élucider la question de savoir si nos résultats concernant la convexité de $\log M_{\alpha \beta}$ subsistent dans le triangle (39), ou même dans tout le carré $\mathrm{o} \leqq \alpha \leqq \mathrm{I}, \mathrm{O} \leqq \beta \leqq \mathrm{I}$. Malheureusement, c'est bien peu de choses que je pourrai dire sur ce sujet. J'espère pouvoir y revenir bientôt.

Tout d'abord, on voit très simplement que, dans le cas particulier où tous les $a_{j k}$ sont positifs ou nuls, il y a convexité dans tout le carré et même dans tout le plan.

En effet, soient $t_{1}$ et $t_{2}$ des nombres positifs tels que $t_{1}+t_{2}=1$. Posons $\alpha=t_{1} \alpha_{1}+t_{z} \alpha_{2}, \beta=t_{1} \beta_{1}+t_{2} \beta_{2}$ et désignons par $\left(x_{j}\right)$ et $\left(y_{k}\right)$ des systèmes positifs satisfaisant aux inégalités

$$
\sum_{j=1}^{m} x_{j}^{\frac{1}{\alpha}} \leqq \mathrm{I} ; \quad \sum_{k=1}^{m} y_{k}^{\frac{1}{\beta}} \leqq \mathrm{I}
$$

Il vient alors par l'inégalitẹ de Hölder 
Sur les maxima des formes bilinéaires et sur les fonctionnelles linéaires.

$$
\begin{gathered}
\sum \sum a_{j k} x_{j} y_{k}=\sum \sum a_{j k}{ }^{t_{1}+t_{2}} x_{j}{ }^{\frac{1}{\alpha}\left(t_{1} \alpha_{1}+t_{2} \alpha_{2}\right)} y_{k} \widetilde{\frac{1}{\beta}}^{\frac{1}{\left.t_{1} \beta_{1}+t_{2} \beta_{2}\right)}} \leqq \\
\leqq\left(\sum \sum a_{j k} x_{j}^{\frac{\alpha_{1}}{\alpha}} y_{k}^{\frac{\beta_{1}}{\beta}}\right)^{t_{1}}\left(\sum \sum a_{j k} x_{j}^{\frac{\alpha_{2}}{\alpha}} y_{k}^{\frac{\beta_{2}}{\beta}}\right)^{t_{2}} .
\end{gathered}
$$

En observant maintenant que les systèmes

$$
x_{j}^{\prime}=x_{j}^{\frac{\alpha_{1}}{\alpha}}, \quad y_{k}^{\prime}=y_{k}^{\frac{\beta_{1}}{\beta}} ; \quad x_{j}^{\prime \prime}=x_{j}^{\frac{\alpha_{2}}{\alpha}}, \quad y_{k}^{\prime \prime}=y_{k}^{\frac{\beta_{3}}{\beta}}
$$

satisfont à des inégalités analogues à (43), les exposants respectifs étant $\frac{\mathrm{I}}{\alpha_{1}}, \frac{\mathrm{I}}{\beta_{1}}$ $\frac{\mathrm{I}}{\alpha_{2}}, \frac{\mathrm{I}}{\beta_{2}}$, notre remarque se trouve établie.

38. Le fait presqu'évident qui précède admet des applications assez intéressantes et remplace dans bien des cas le théorème I beaucoup plus caché. Considérons à titre d'exemple l'application suivante.

Soient, pour la forme bilinéaire $A(x, y)=\sum_{j=1}^{m} \sum_{k=1}^{n} a_{j k} x_{j} y_{k}, \quad \sum_{k=1}^{n}\left|a_{j k}\right| \leqq G$ $(j=\mathrm{I}, 2, \ldots m), \quad \sum_{j=1}^{m}\left|a_{j k}\right| \leqq H(k=\mathrm{I}, 2 \ldots n)$.

On aura alors $M_{1,0} \leqq G, M_{0,1} \leqq H$ et par conséquent $M_{\alpha, 1-\alpha} \leqq G^{\alpha} H^{1-\alpha}$. En particulier $M_{\frac{1}{2}, \frac{1}{2}} \leqq \sqrt{G H}$, inégalité due à Frobenios et à M. I. Schor. ${ }^{1}$

L'extension aux opérations bilinéaires et, en particulier, aux intégrales doubles de la forme $\iint K(x, y) f(x) g(y) d x d y$ est immédiate.

39. Le fait établi au $\mathrm{n}^{\circ} 37$ et ses généralisations évidentes embrassent à peu près tout ${ }^{2}$ ce que je sais dire d'affirmatif sur la convexité dans des domaines plus étendus que le triangle (2). Voici maintenant ce qui est de caractère négatif.

Considérons la forme bilinéaire réelle $A(x, y)=X_{1} y_{1}+X_{2} y_{2}$ où $X_{1}=x_{1}+x_{2}$ et $X_{2}=x_{1}-x_{2}$. Etudions le maximum de cette forme en supposant les variables de rester réelles et de varier sous les conditions

${ }^{1}$ I. ScHUR, Bemerkungen zur Theorie der beschränkten Bilinearformen mit unendlich vielen Veränderlichen, Journ. f. Math. t. 140 (I9II), p. 1-28, voir, en particulier, p. 6.

2 On pourrait encore y ajouter le fait presqu'évident que, pour des $a_{j k}$ quelconques, il y a convexité dans tout le plan sur les droites $\alpha=$ const. et $\beta=$ const. 


$$
\left|x_{1}\right|^{\frac{1}{\alpha}}+\left|x_{2}\right|^{\frac{1}{\alpha}} \leqq \mathrm{I} ; \quad\left|y_{1}\right|^{\frac{1}{\beta}}+\left|y_{2}\right|^{\frac{1}{\beta}} \leqq \mathrm{I}
$$

Cela revient à considérer le maximum de

$$
\left(\left|X_{1}\right|^{\frac{1}{1-\beta}}+\left|X_{2}\right|^{\frac{1}{1-\beta}}\right)^{1-\beta}
$$

sous la première des conditions (44).

On voit facilement que le logarithme de ce maximum n'est pas convexe sur le segment limité par les points $(0,0)$ et $\left(\frac{1}{2}, \frac{1}{2}\right)$. En effet, on a $M_{0,0}=M_{0,1}^{*}=2$, $M_{\frac{1}{2}, \frac{1}{2}}=M_{\frac{1}{2}, \frac{1}{2}}^{*}=2^{\frac{1}{2}}$. D'autre part, en calculant le rapport

$$
\left(\left|X_{1}\right|^{\frac{1}{1-\alpha}}+\left|X_{2}\right|^{\frac{1}{1-\alpha}}\right)^{1-\alpha}:\left(\left|x_{1}\right|^{\frac{1}{\alpha}}+\left|x_{2}\right|^{\frac{1}{\alpha}}\right)^{\alpha}
$$

pour un système arbitraire différent des systèmes $x_{1}= \pm x_{2}$ et des systèmes où l'une des variables s'annule, on trouve une valeur plus grande que

$$
2^{1-\alpha}=\left(M_{0,0}\right)^{\frac{\frac{1}{2}-\alpha}{2}}\left(M_{\frac{1}{2}}, \frac{1}{2}\right)^{\frac{\frac{\alpha}{1}}{2}}
$$

Cela se roit aussi sans calcul, en observant que pour $\alpha=0$ et pour $\alpha=\frac{1}{2}$ - et seulement pour ces valeurs-là - le rapport (46) ne change pas avec les variables, et que le logavithme du minimum du rapport est concave, ce dernier fait ressortant du théorème $I$ appliqué à la forme réciproque. Le minimum et le maximum coïncidant pour les extrémités du segment, le logarithme du maximum ne pourra être couvexe.

Par conséquent, si l'on se restreint, pour les formes réelles, aux valeurs réelles des variables, nos théorèmes ne tiennent plus dans le triangle (39).

40. Dans l'exemple qui précède, le vrai maximum est fourni par des systèmes complexes tels que $x_{1}: x_{2}=\mathrm{I}: \pm i$. La valeur du logarithme du maximum est donnée par la fonction $\log 2$. $\left(\frac{3}{2}-2 \alpha\right)$; c'est-à-dire que ce logarithme est une fonction linéaire et alors une fonction convexe dans le sens que nous avons attribué à ce mot. On voit aussi sans peine que, dans cet exemple, le logarithme du vrai maximum est convexe dans tout le carré $0 \leqq \alpha \leqq I, 0 \leqq \beta \leqq I$. 
Sur les maxima des formes bilinéaires et sur les fonctionnelles linéaires. $\quad 497$

Nos considérations font voir que si, dans le cas général, la question de la validité de nos théorèmes sera à résoudre par l'affirmative pour tout le carré - ce qui d'ailleurs parait peu probable - les relations (12), desquelles nous avons déduit nos théorèmes pour le triangle (2), ne suffiront certainement pas pour fournir ce résultat. En effet, ces relations tiennent évidemment aussi pour les systèmes qui fournissent le maximum d'une forme bilinéaire réelle, quand on se restreint aux valeurs réelles des variables. 